\title{
Robust Memory Gradient Blind Equalization Algorithm Based on Error Sign Decision
}

\author{
Fangfang HAN \\ Urumqi Station \\ State Radio Monitoring Center \\ Urumqi, Xinjiang,China \\ hanfangfang0993@163.com
}

\author{
Guoqiang XU \\ Urumqi Station \\ State Radio Monitoring Center \\ Urumqi, Xinjiang,China \\ xuguoqianghebei@163.com
}

\begin{abstract}
As CMA blind equalization algorithm based on stochastic gradient descend method has slow convergence rate and big steady state residual error, a robust memory gradient blind equalization algorithm based on error sign decision was proposed. Compared with stochastic gradient algorithm, memory gradient algorithm can make full use of the iteration gradient information of current and last step to accelerate convergence speed and effectively avoid the error convergence algorithm to a certain extent. However, transient noise interference may result in inconsistent direction of current iteration gradient information and last step that may cause the algorithm unstable, if the iteration error sign decision of current and last step is consistent then adopts memory gradient stochastic gradient descent algorithm, this method can ensure the robustness of memory gradient algorithm .The computer simulation results prove the validity of the algorithm.
\end{abstract}

Keywords- Blind Equalization; Memory Gradient; CMA; Error Sign

\section{INTRODUCTION}

In digital communication system, multipath propagation leads to severe inter symbol interference (ISI),equalization is an effective technical to eliminate ISI. But training sequence need to be sent periodically in traditional adaptive equalization techniques to capture and track the characteristics of the channel and compensate it. In many communication situations, such as multicast communication, battlefield information interception and other fields, no training sequence can be used, and this will wastes some channel bandwidth , affect the efficiency of communications. Blind equalization originally proposed by Sato et al can realize the communication channel compensation without training sequence, elimination ISI caused by multipath timevarying method [1], improve the bandwidth utilization efficiency and the quality of communication ,it has important significance in modern digital communication. The field of blind channel equalization has gained increasing attention since it was proposed and a mass of theories and methods have been achieved. The most commonly used blind adaptation algorithm is the Constant Modulus Algorithm (CMA) for it is simple and it's stable convergence [2], as CMA is one of the gradient descent algorithms, it has slow convergence speed, and because the cost function is not convex, the algorithm is easy to fall into local minimal value. Some improved algorithms such as the quasi-Newton method and conjugate gradient method, although to a certain extent can improve the convergence speed, but it requires high computational complexity, $t$ there is a conflict between he algorithm performance and computation complexity ,so it is not easily applied to engineering. Like conjugate gradient algorithm, memory gradient algorithm does not require the storage and computation of Hessian matrix [3], and is particularly suitable for solving large-scale optimization problems, in blind equalization, more conducive to real-time applications. Considering transient noise interference may result in inconsistent direction of current iteration gradient information and last step that may cause the algorithm unstable, if the iteration error sign decision of current and last step is consistent then adopts memory gradient algorithm to update the equalizer weight, else still adopts the SG descent algorithm, this method can ensure the robustness of MG algorithm .The computer simulation results prove the validity of the algorithm.

II. THE BASIC PRINCIPLE OF CMA BLIND EQUALIZATION

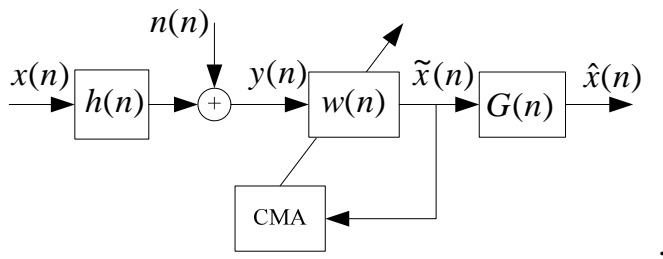

Figure 1. Block diagram of CMA blind equalization

Fig.1shows the block diagram of CMA blind equalization [4], The input signal $\boldsymbol{x}(n)$ transmits through the unknown channel $\boldsymbol{h}(n)$ adding Gauss white noise $\boldsymbol{n}(n)$, and then we can get the observation signal $\boldsymbol{y}(n)$ before the blind equalizer. $\tilde{x}(n)$ is the output signal of the blind equalizer, and $\hat{\boldsymbol{x}}(n)$ is the decision symbol of .Blind equalization can recover the input signal $\boldsymbol{x}(n)$ only rely on the observation signal $\boldsymbol{y}(n)$. CMA algorithm is one of the Godard algorithms with the parameter $p=2$, which is the special case of Bussgang blind equalization with the most widely used. The cost function of CMA is [5] 


$$
J_{C}=\frac{1}{2}\left[\left.\tilde{x}(n)\right|^{2}-R_{C M}\right]^{2}
$$

where $R_{C M}$ is the constant modulus can compute by

$$
R_{C M}=\frac{E\left(|\tilde{x}(n)|^{4}\right)}{E\left(|\tilde{x}(n)|^{2}\right)}
$$

According to the stochastic gradient descent algorithm, Minimum cost function of CMA is given by [3]

$$
\begin{gathered}
w(n+1)=w(n)+\frac{\partial J_{C}}{\partial w(n)} \\
J_{C}=\frac{1}{2}\left[\left|w(n) y^{T}(n)\right|-R_{C M}\right]^{2} \\
\frac{\partial J_{C}}{\partial w(n)}=2\left[|\tilde{x}(n)|^{2}-R_{C M}\right] \tilde{x}(n) y^{*}(n)
\end{gathered}
$$

where the symbol * denotes conjugate operation. Error function of CMA blind equalization algorithm $e_{C}(n)$ is

$$
e_{C}(n)=2\left[|\tilde{x}(n)|^{2}-R_{C M}\right]
$$

weight update of CMA blind equalization algorithm is given by [7]

$$
w(n+1)=w(n)+\mu e_{C}(n) \tilde{x}(n) y^{*}(n)
$$

where $\mu$ is the step size to modify each iteration of gradient updating size, bigger step value has a faster convergence speed with big steady residual error, but smaller step value can get smaller steady residual error convergence with slow convergence speed .

\section{ROBUST MEMORY GRADIENT BLIND EQUALIZATION ALGORITHM}

Traditional CMA blind equalization using stochastic gradient descent algorithm for network weights and parameters update, although the structure is simple and computation complexity is low ,but slow convergence may lead to generate seesaw phenomenon, as the convex cost function, it is difficult to converge to the global optimal solution, the stochastic gradient algorithm, weighted iterative updating formula can be written as (7), where $g(n)$ is gradient function given by (8).

$$
g(n)=-\frac{\partial J_{C}(n)}{\partial w(n)}
$$

The innovation of the weight-vector in SG algorithm is given by

$$
w(n+1)=w(n)+\mu g(n)
$$

Eq.9 can be also rewritten as

$$
w(n+1)=w(n)+\mu H(n) g(n)
$$

Then improved algorithm is quasi Newton algorithm, where $H(n)$ approximately equal to $\nabla^{2} J_{D}{ }^{-1}$ or $\nabla^{2} J_{D}{ }^{-1}$, Quasi Newton method has a faster convergence rate in certain conditions, but it requires the computation and storage of Hessen matrix in each iteration, the computational complexity is high, quasi Newton algorithms mostly limited in real system. Guangrong Yan et al centered on quasi Newton algorithm's application in blind equalization, and has achieved admirable equalization effect, but this method requires the information of the target function's two order derivative, that require the objective function for the real and imaginary parts of the partial derivatives exist, this condition may not be able to meet strictly in practical communication systems. Conjugate gradient algorithm is a SG algorithm on the basis of an efficient algorithm each iteration through the memory of the previous step iterative information to generate the next iteration, thereby generating conjugate gradient direction, standard conjugate gradient algorithm weights iterative basic methods such as type (11)

$$
w(n)=w(n)+\mu(n) d(n)
$$

where

$$
d(n)=\left\{\begin{array}{cc}
-g(n) & n=1 \\
-g(n)+\beta(n) g(n-1) & n \geq 2
\end{array}\right.
$$

Different values of $\beta(n)$, will give different forms of conjugate gradient algorithms, such as FR, PR, HS conjugate gradient algorithm.

$$
\begin{gathered}
\beta^{F R}(n)=\frac{\|g(n)\|^{2}}{\|g(n-1)\|^{2}} \\
\beta^{P R}(n)=\frac{g^{T}(n)(g(n)-g(n-1))}{\|g(n-1)\|^{2}} \\
\beta^{H S}(n)=\frac{g^{T}(n)(g(n)-g(n-1))}{d^{T}(n-1)(g(n)-g(n-1))}
\end{gathered}
$$

If it does not take the restart strategy, conjugate gradient algorithm the most linear convergence, therefore, the conjugate gradient algorithm in nonlinear objective function 
is generally not have global convergence, as objective function of wavelet neural network blind equalization is constant modulus blind equalization cost function, the objective function is the multi-dimensional non-convex, therefore, it is very difficult to ensure that the algorithm converges to the global optimal use of conjugate gradient algorithm. MG algorithm is used in order to guarantee that the algorithm convergence to the global optimum ,As the conjugate gradient algorithm, memory gradient algorithm does not require the storage and calculation of the matrix, and is particularly suitable for solving large-scale optimization problems, in blind equalization, more conducive to real-time applications. Memory gradient algorithm weight iterative formula is

$$
w(n)=w(n)+\mu(n) d(n)
$$

where

$$
d(n)=\left\{\begin{array}{cc}
-g(n) & n=1 \\
-[(1-\beta(n)) g(n)+\beta(n) g(n-1)] & n \geq 2
\end{array}\right.
$$

where $\beta(n)$ is given as follow

$$
\beta(n)=\frac{\gamma\|g(n)\|^{2}}{\|g(n)\|^{2}+\left|g^{T}(n) g(n-1)\right|}
$$

where $\gamma \in(0,1)$, as can be seen, memory gradient algorithm made full use of to the previous iterative gradient information, by setting the parameter $\gamma$ to control the memory information in an iterative process of effect size. In the actual communication system, transmission signals are inevitably subject to noise effects, thus, that may directly leads to the instantaneous gradient instability cannot guarantee the gradient direction, reflected in the instabilities of $\beta(n)$. To further ensure the reliability of MG algorithm blind equalization, a robust memory gradient (MG)blind equalization algorithm based on error sign decision was proposed, the idea of the algorithm is that ,if iteration error sign decision of current and last step is consistent ,and it illustrates the algorithm convergence in the same direction ,then adopts MG algorithm to update the equalizer weight, else that means the gradient information of current and last step is not relevant, still adopts the SG descent algorithm, that can be controlled by set parameter $\gamma$.

$$
\gamma=\left\{\begin{array}{cc}
0 & \operatorname{sgn}(e(n) e(n-1))>0 \\
\gamma_{\text {int }} & \operatorname{sgn}(e(n) e(n-1))<0
\end{array}\right.
$$

\section{COMPUTER SIMULATION}

In order to validate the performance of robust memory gradient (MG)blind equalization algorithm based on error sign decision ( SG-FG-CMA ), CMA algorithm and memory gradient algorithm for blind equalization algorithm (FG-CMA) were simulated in the same simulation conditions, define residual inter-symbol interference ISI to evaluate performance of algorithms [6].

$$
I S I=\frac{\sum_{i}\left|C_{i}\right|^{2}-\left|C_{i}\right|_{\max }^{2}}{\left|C_{i}\right|_{\max }^{2}}
$$

In Eq.20, C is the combined impulse response of the channel and equalizer. Channel is mixed phase channel model $\mathrm{h}=[0.3132,-0.1040,0.8908,0.3143]$, QPSK signal is used in the simulation as the input signal ,the weight length of the filter is 41 . central tap is initialized to 1,step size $\mu_{c}=0.0008$

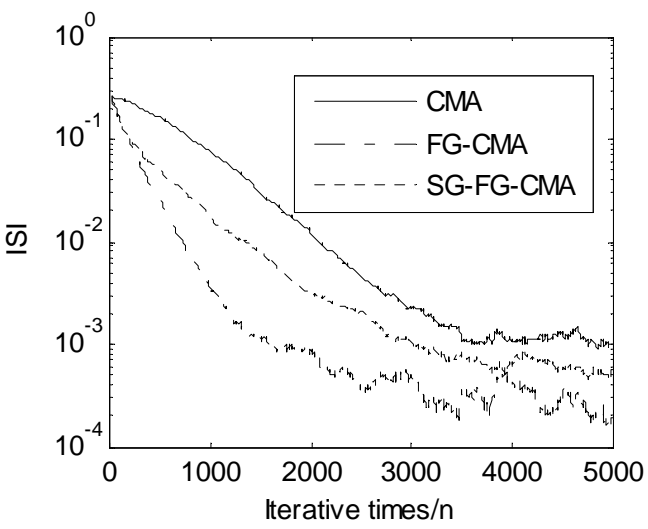

Figure 2. ISI convergence curves $(\mathrm{SNR}=21.3 \mathrm{~dB})$

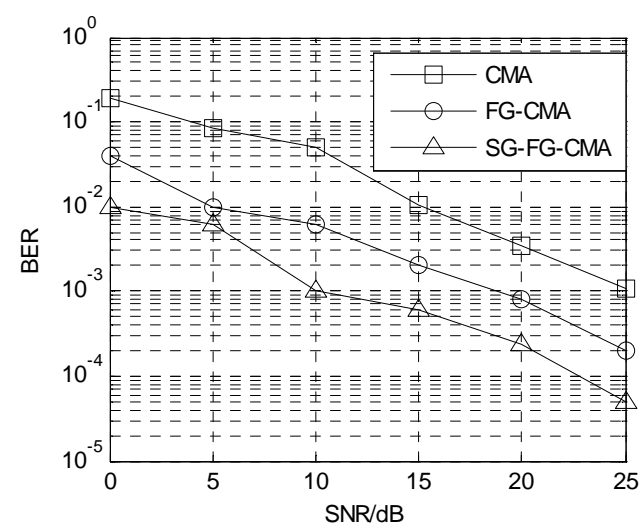

Figure 3. BER under different SNR

As can be seen from Fig.2 the proposed SG-FG-CMA has faster convergence speed and higher convergence precision compared with CMA and FG-CMA. From Fig.3, 
we can see SG-FG-CMA has low bit error rate, it proves the robust equalization performance of the algorithm under different signal-to-noise ratio (SNR).

\section{CONCLUSION}

Robust memory gradient blind equalization algorithm based on error sign decision is proposed by given a detailed analysis of memory gradient algorithm. Equalizer weight updated by stochastic gradient algorithm and memory gradient algorithm depend on the iteration error sign decision of current and last step is consistent or not in SG-FG-CMA, and that effectively improve the speed of convergence and convergence precision and it has a robust equalization performance under different SNR. Compared with the conjugate gradient algorithm and quasi-Newton algorithm memory gradient algorithm has low computational complexity, so it has practicality value in project.

\section{REFERENCES}

[1] Xie Ning, Hu Hengyun, Wang Hui. A new hybrid blind equalization algorithm with steady-state performance analysis [J]. Digital Signal Processing: A Review Journal, 2012, 22(2): 233-237.

[2] Niroomand M., Derakhtian M., Masnadi-Shirazi M.A. Steady-state performance analysis of a generalised multimodulus adaptive blind equalisation based on the pseudo Newton algorithm [J]. IET Signal Processing, 2012, 6(1): 14-26.

[3] Xiao Ying, Dong Yu-Hua. Blind Equalization by Wavelet Neural Network with Nonlinear Memory Gradient Algorithm [J]. International Journal of Digital Content Technology and its Applications, 2011, 5(12): 249-258.

[4]Ying Xiao, Zhenxing Li. Wavelet neural network blind equalization improved by modified decorrelation algorithm for underwater acoustic[J]. Journal of Computational Information Systems, 2012, 8(11): 4459-4466.

[5]Ma Lili, Chen Jinguang, Wang Huiran. Performance analysis of the affine projection CM algorithm [J].Journal of Information and Computational Science,2011,8(14): 3275-3280.

[6] RAO Wei, GUO Ye-cai, WANG Sheng-qian, etal. New constant modulus algorithm for blind equalization based on coordinate transformation [J]. Acta Electronica Sinica, 2011, 39(1):7-12. 